\title{
A $0.4-1.4 \mathrm{~V} 24 \mathrm{MHz}$ fully integrated $33 \mu \mathrm{W}, 104 \mathrm{ppm} / \mathrm{V}$ supply-independent oscillator for RFIDs
}

\author{
Valentijn De Smedt, Wim Dehaene and Georges Gielen \\ Katholieke Universiteit Leuven, Dept. Elektrotechniek, afd. ESAT-MICAS \\ Kasteelpark Arenberg 10, B-3001 Heverlee, Belgium \\ Email: valentijn.desmedt@esat.kuleuven.be
}

\begin{abstract}
In RFID-tags with pulse-based UWB communication, accurate supply-independent low-power oscillators are required. The $24 \mathbf{M H z}$ oscillator presented was realized in a $130 \mathrm{~nm}$ CMOS technology. It has an ultra-low supply voltage dependency of $104 \mathbf{~ p p m} / \mathrm{V}$ over a voltage range of $1.4 \mathrm{~V}$ to $0.4 \mathrm{~V}$. This was achieved by the use of two nested ultralow-power voltage regulators and a novel circuit technique based on the attraction of two oscillator frequencies. The mean power consumption is $33 \mu \mathbf{W}$ over the $1 \mathrm{~V}$ voltage span. No external biasing and no trimming or calibration was used.
\end{abstract}

\section{INTRODUCTION}

One of the main problems in RFID-tags is the power supply. In RF-powered RFID-tags, the power is provided by energy scavenging, while the scavenged energy is stored in a capacitor (figure 1, [1]). During data transmission or computations on the tag, the capacitor is discharged and the supply voltage will drop (T-active in figure 1). Since most of the (digital) circuitry on a tag can run at low speed, this voltage drop and low supply voltage should not be a problem. However, in a lot of applications RFID-tags need to be localizable. This can be achieved by measuring the time of arrival of UWB-pulses [2]. To be able to receive the UWB-pulses, they must be transmitted at an accurate rate [3]. This requires a stable low frequency reference. The lower the minimum operating voltage (Vmin) and the power consumption of the circuitry, the longer the tag will be able to work.

This paper presents the design of an ultra-low-power, supplyindependent clock reference. Section II describes the problem of the changing supply voltage for different oscillator topologies. Afterwards, the proposed low-power oscillator is discussed in section III. Next, section IV discusses the used voltage and current regulator. In section $\mathrm{V}$ some measurement results are presented and compared to state of the art designs. Finally, some conclusions are drawn in section VI.

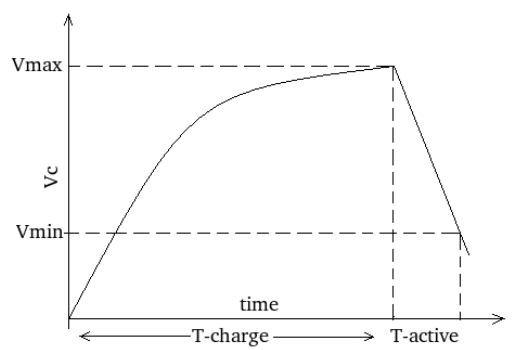

Fig. 1. Typical charge and discharge characteristic of the supply capacitor on an RFID-tag. Vc is the voltage on the supply capacitor. The lower the minimum operating voltage (Vmin) and power consumption of the tag, the longer it will be able to work.

\section{THE OSCILlator TOPOLOGY}

To make a supply-independent time reference, there are two possibilities: the first is to make the time reference itself independent of the supply voltage, for instance based on voltage-independent RC or LC resonators. Another possibility is to build a voltage reference which serves as a stable voltage source to supply a low-voltage, possibly voltage-dependent, time reference. Both possibilities are discussed.

\section{A. Voltage-independent Time References}

The problem with a varying supply voltage is that the transistor properties change with the supply voltage. Switches become slower when the supply voltage drops and amplifier properties such as the transconductance and the output resistance are sensitive to the changing dc biasing conditions. These changing transistor parameters have an influence on the oscillation frequency. One should therefore try to make a time reference which is independent of the active components.

In the case of a harmonic oscillator, an amplifier and a tuned feedback network are used. When the Barkhausen criterion is fulfilled, i.e. the complex loop gain is equal to one, a stable oscillation is observed. To eliminate the influence of the active components, an ideal, voltage-independent amplifier is needed or the influence of the amplifier on the oscillation frequency has to be negligible. The Qfactor, (1), is a measure for the energy efficiency and the frequency selectivity of a resonator [4]. When a high-Q resonator, for instance a crystal or an LC-tank, is used, little energy is added by the amplifier to preserve an oscillation. In this way, the changing transistor parameters have a negligible effect on the oscillation frequency [5]. However, in low-power applications the use of an integrated LC-resonator is not suitable due to its high-frequency behavior. Also, the use of external components, i.e. a crystal, is not advantageous in this kind of applications.

$$
Q=\omega \cdot \frac{E_{\text {stored }}}{P_{\text {loss }}}=2 \cdot \pi \cdot \frac{E_{\text {stored }}}{E_{\text {Dissipated-per-Cycle }}}
$$

An alternative is to use an RC oscillator. By using the definition of the Q-factor (1) one can easily prove that the Q-factor of an RC feedback network cannot be higher than 0.5. Because of the low Q-factor, the energy provided by the amplifier should be rather high to preserve a stable oscillation. The influence of the amplifier on the oscillation frequency is not negligible anymore and an amplifier independent of the changing transistor parameters must be used. However, when the supply voltage drops below $0.5 \mathrm{~V}$, this seems to be hardly possible. In low-voltage operation, a high output impedance cannot be obtained because of the low drain-source voltage $V_{D S}$. Cascoding can not be used either. Neither can a low impedance be obtained because of the limited supply current or power consumption.

Instead of a harmonic oscillator, a relaxation oscillator can be used. These oscillators always depend on one or more non-linear components, which are in this case transistors. When the voltage drops, transistors change and switches become slower. In this way, 


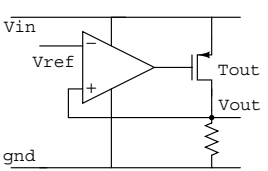

Fig. 2. An LDO regulator typically consists of a voltage reference, an output transistor and a feedback amplifier.

the frequency is affected by the changing supply voltage, even if the main frequency-determining components (mostly a capacitor and a resistive element) are kept constant. It can be concluded that it is extremely difficult to make supply independent oscillators.

\section{B. Regulating the Supply Voltage}

Another way to build a voltage-independent time reference is by using a low dropout (LDO) voltage regulator. Typically a regulator consists of a voltage reference, an output transistor and a feedback amplifier (figure 2). The output resistance of the regulator is equal to the $r_{0}=V_{E} \cdot L / I$ of the output transistor $T_{O U T}$ divided by the loop gain. Note that this output resistance is frequency-dependent. Since $V_{D S}$ changes drastically as a function of the power supply, the output resistance $r_{0}$ changes and also the gain of the feedback amplifier will be voltage-dependent. A high loop gain or a low $r_{0}$ is required to keep the output resistance low and diminish the influence of the regulator on the oscillator. This, however, will lead to a high power consumption. Still, there is an option to cancel out the influence of the regulator's changing output resistance. When a constant current is drawn from the regulator and the output voltage is kept constant, the output resistance is no longer influential. The challenge now becomes to build an oscillator which has a constant power consumption over time. In the case of a relaxation oscillator, a constant power consumption is rather difficult to achieve due to the non-linear switching. Harmonic oscillators on the other hand can be built with only linear components. Some differential topologies with a constant power consumption are available [6], [7]. Therefore, this approach is used to build a supply-independent time reference. The proposed solution will be discussed next.

\section{THE PROPOSED OSCILLATOR}

The oscillator is based on a well-known RC oscillator structure: the Wienbridge oscillator (figure 3, [6]). The oscillation frequency of this oscillator is equal to:

$$
f=\frac{1}{2 \cdot \pi \cdot R \cdot C}
$$

As explained before, amplifiers with a high gain and a low output impedance are difficult to realize in low-power and low-voltage operation. Therefore, in the proposed solution, the forward amplifier is a cascade of two inverting common-source amplifiers and the resistors in the feedback network are equal to the output resistance of the amplifiers (figure 4). The resistor to the ground in the feedback network is implemented by a dummy amplifier. Both the output resistance and capacitance of these low-power amplifiers become a part of the feedback network. For the biasing of the circuit, a replica, biased by the regulators (section IV), is used. As discussed in section II-B, the output resistance of the regulators will influence the oscillation frequency if the oscillator has a time varying power consumption.

\section{A. Coupling Two Oscillators}

To overcome the problem of the changing regulator's output resistance, a differential structure is proposed: a combination of

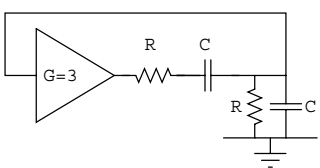

Fig. 3. The Wienbridge oscillator is a harmonic RC oscillator consisting of an amplifier with a gain of 3 and a tuned RC feedback network.

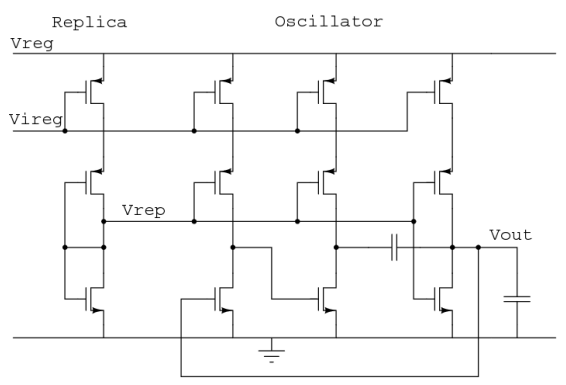

Fig. 4. The used Wienbridge oscillator. The resistors in the feedback network are implemented by the output impedance of the amplifiers. $V_{\text {reg }}$ and $V_{\text {ireg }}$ are biasing voltages both delivered by the regulator circuitry.

two oscillators oscillating in opposite phase. In [6] this differential oscillating structure is built by cascading two amplifiers and two RC feedback networks. In the ideal case, this structure is completely differential. However, any mismatch between the amplifiers or feedback networks will disturb the phase shift of $180^{\circ}$ between the two oscillators. Using Simpson's formula, one can easily prove that, for a small deviation, the variation on the drawn current is proportional to the phase shift, $2 \cdot \delta$, between the oscillators:

$$
\begin{aligned}
\sin (\omega \cdot t+\delta)-\sin (\omega \cdot t-\delta) & =2 \cdot \sin (\delta) \cdot \cos (\omega \cdot t) \\
& \sim 2 \cdot \delta \cdot \cos (\omega \cdot t)
\end{aligned}
$$

Since in this design a cascade of two inverting amplifier stages is used, it is possible to couple the in-phase nodes of the two oscillators oscillating in opposite phase with coupling capacitors (figure 5). To draw a constant current through the biasing current sources, each current source is shared by two differentially oscillating amplifiers. The coupling capacitors $C_{C}$ are in the order of $20 \mathrm{fF}$ which makes careful layout and parasitic extraction necessary. If any mismatch appears between the two oscillators, the resulting oscillation frequency is between the two individual oscillator frequencies. However, both oscillators are always oscillating in opposite phase and the current consumption stays constant over time. Compared to the differential oscillator proposed in [6], the ripple on the drawn current is almost a factor 2000 lower for a capacitor mismatch of $10 \%$.

\section{B. Operating in Weak-Inversion}

Due to the low-power and low-voltage operation, the transistor devices in the amplifiers have a very low $I_{D S}$ and $V_{G S}-V_{T h}$ which makes them operate in weak inversion rather than in strong inversion. In this operating region, $I_{D S}$ has an exponential behavior as a function of $V_{G S}$ instead of the quadratic behavior in the saturation region. The following equation describes the behavior of a transistor in weak inversion, note that the dependency of the transconductance $\left(g_{m}\right)$ on $V_{G S}$ is also exponential [7]:

$$
g_{m}=\frac{I_{D 0}}{n k T / q} \cdot \frac{W}{L} \cdot \exp \left(\frac{V_{G S}}{n k T / q}\right)
$$

where $I_{D 0}$ and $n$ are technology constants, $T$ is the absolute temperature, $k$ is the Boltzman constant and $q$ is the charge of 


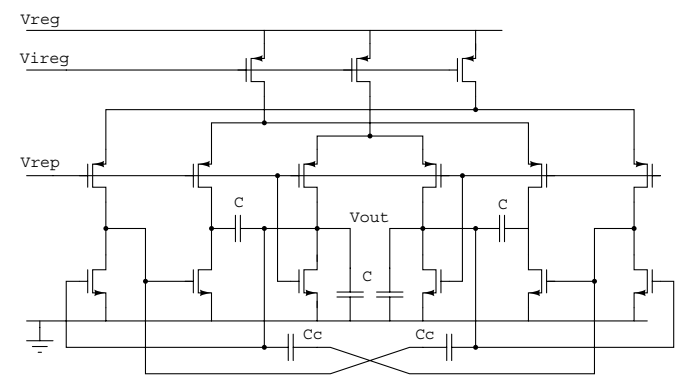

Fig. 5. The two oscillators are coupled in opposite phase by the coupling capacitors $C_{C}$. Note that the current sources of the differentially oscillating amplifiers are shared to draw a constant current over time. The biasing voltage $V_{r e p}$ is delivered by an amplifier replica, $V_{\text {reg }}$ and $V_{\text {ireg }}$ are biasing voltages both delivered by the regulator circuitry.

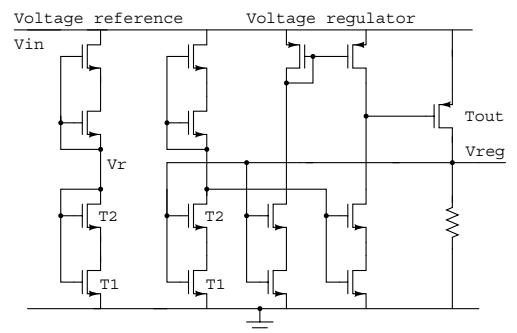

Fig. 6. The voltage regulator is based on the voltage reference on the left. The output voltage is determined by the threshold voltages of $T_{1}$ and $T_{2}$.

a single electron. An important drawback of a transistor in weak inversion is the sensitivity of the dc biasing to transistor mismatch and temperature. As for the problem of transistor mismatch, replica biasing is mentioned to overcome this problem. Another technique to improve the matching is splitting every transistor into four parts and layout them in a way that the current flows in four directions. In this way the systematic mismatch is reduced. The temperature dependency on the other hand is not a problem for this application since the temperature stays more or less constant during the short operation period.

Another drawback of ultra-low-power circuits is the noise. Due to the high impedance on the nodes in the circuit, a lot of noise is observed. This noise contributes to the noise at the output and is translated to phase noise or jitter. However, even in RFID applications where the UWB-pulses are used for localization, the distance measurement is not disturbed by the jitter of the oscillator [3], [2].

\section{THE LDO REgULATOR}

To obtain a constant supply voltage for the oscillator, a voltage regulator is needed. The used regulator is shown in figure 6 and is based on the reference circuit on the left [8]. The output voltage of the reference, $V_{r}$, is determined by the threshold voltages of $T_{1}$ and $T_{2}$. The leakage current of the NMOS transistors in cutoff is less than $150 \mathrm{nA}$. When the supply voltage varies from $1.4 \mathrm{~V}$ to $0.4 \mathrm{~V}$ the output drops from $337.4 \mathrm{mV}$ to $334.2 \mathrm{mV}$. Since this variation is still $1 \%$, a voltage and a current regulator are combined to further increase the stability.

\section{A. The Voltage Regulator}

One of the advantages of the used LDO regulator is the voltage reference which is part of the feedback amplifier (figure 6). Due to the extremely low current in the regulator branches, the dc loop gain of this regulator is around $114 \mathrm{~dB}$. The gain-bandwidth of the feedback

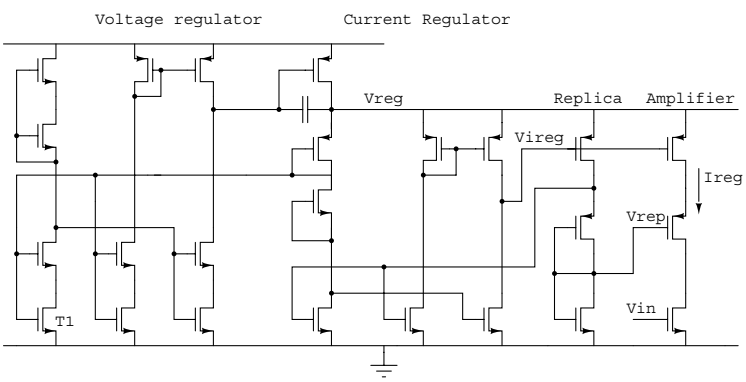

Fig. 7. Two regulators are used to provide a stable output voltage and current to the oscillator. The current regulator is directly connected to a replica of the amplifier used in the oscillator. The output current of the regulator only changes $0.02 \%$ over the $1 \mathrm{~V}$ voltage drop.

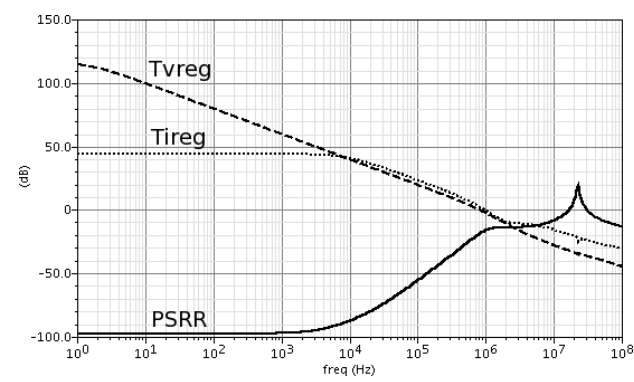

Fig. 8. The simulated loop gain of the regulators (Tvreg and Tireg) and the power supply rejection ratio (PSRR) at the output of the oscillator. The supply voltage was $0.9 \mathrm{~V}$.

system is around $820 \mathrm{kHz}$. Similar to the voltage reference itself, the output voltage of the regulator only drops $3 \mathrm{mV}$ with an input voltage drop from $1.4 \mathrm{~V}$ to $0.4 \mathrm{~V}$. Figure 7 shows how the current regulator is connected to the output of the voltage regulator.

\section{B. The Current Regulator}

The current regulator has the same topology as the voltage regulator. Because of the lower available supply voltage, the cascodes are left out. This regulator is directly connected to a replica of the amplifier used in the oscillator. In this way the current regulator controls the biasing current sources in the oscillator. The dc loop gain in the current regulator is equal to $43.3 \mathrm{~dB}$ and the gain-bandwith is $978 \mathrm{kHz}$. Due to the combination of two regulators, the amplifier's biasing current varies less than $0.02 \%$ over the $1 \mathrm{~V}$ supply voltage drop. To make the feedback loop of the voltage regulator stable, a Miller capacitor [7] is used to connect the gate of the output transistor to the output (figure 7). The loop gain of the regulators (Tvreg and Tireg) and the power supply rejection ration (PSRR) at the oscillator's output are plotted in figure 7 . These graphs were simulated at a supply voltage of $0.9 \mathrm{~V}$, around $0.4 \mathrm{~V}$, however, the loop gain drops and the PSRR vanishes. Also, in these regulators most of the transistors are biased in weak inversion. The benefit of this is the low power consumption and the high dc gain in the feedback loop.

\section{Measurement Results}

The oscillator was designed in a triple-well $0.13 \mu \mathrm{m}$ CMOS technology with low-threshold devices and $1.5 \mathrm{fF} / \mu \mathrm{m}^{2}$ MiM-capacitors. A chip microphotograph is shown in figure 9; the active area measures $200 \mu \mathrm{m} \times 150 \mu \mathrm{m}$. Due to the mismatch between the devices in weak inversion (section III-B), upscaling the devices was necessary. A point-symmetric layout, with every current flowing in four directions, was drawn to improve the matching. Twelve samples of the same 
TABLE I

COMPARISON TO THE STATE OF THE ART

\begin{tabular}{|c|c|c|c|c|c|c|c|c|}
\hline Ref. & $\begin{array}{c}\text { Tech. } \\
{[\mu \mathrm{m}]}\end{array}$ & Oscillator & $\begin{array}{c}\mathrm{f} \\
{[\mathrm{MHz}]}\end{array}$ & $\begin{array}{c}\text { V Sens. } \\
{[\mathrm{ppm} / \mathrm{V}]}\end{array}$ & $\begin{array}{c}\mathrm{I} \\
{[\mu \mathrm{A}]}\end{array}$ & $\begin{array}{c}\text { V-Range } \\
{[\mathrm{V}]}\end{array}$ & $\begin{array}{c}\text { Rel. V-Range } \\
{[\%]^{*}}\end{array}$ & $\begin{array}{c}\text { Trimming/ } \\
\text { Calibration }\end{array}$ \\
\hline \hline This Work & 0.13 & harmonic, RC & 24 & 104 & 37 & $0.4-1.4$ & 111 & no \\
\hline [9], ISSCC09 & 0.18 & relaxation, RC & 14 & $16 \mathrm{e} 3$ & 24 & $1.7-1.9$ & 22 & no \\
\hline [10], SBCCI03 & 0.5 & relaxation, RC & 12.8 & $5.3 \mathrm{e} 3$ & 133 & $2.5-5.5$ & 75 & yes \\
\hline [5], JSSC07 & 0.35 & harmonic, LC & 12 & 38 & $9.5 \mathrm{~mA}$ & $4.5-5.5$ & 20 & yes \\
\hline [11], JSSC06 & 0.25 & ring & 7 & $8.8 e 3$ & 600 & $2.4-2.75$ & 14 & no \\
\hline
\end{tabular}

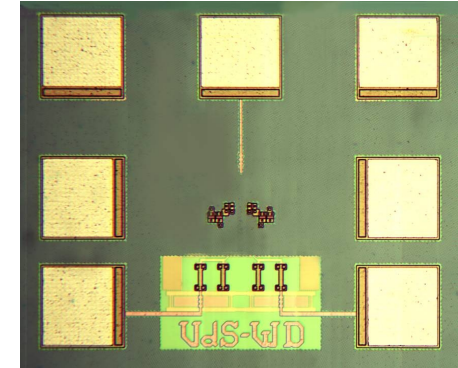

Fig. 9. Chip microphotograph of the proposed oscillator, the active area measures $200 \mu m \times 150 \mu m$.

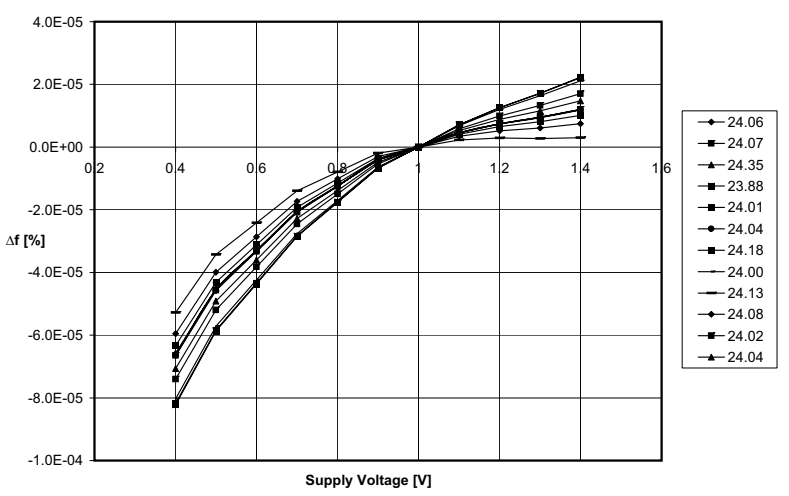

Fig. 10. Relative frequency deviation of the measured samples. On the right, the oscillation frequency of each sample at $1 \mathrm{~V}$ is reported. The maximum frequency variation was $104 \mathrm{ppm}$ over the $0.4 \mathrm{~V}-1.4 \mathrm{~V}$ supply voltage span.

batch were measured and worked over a voltage range of $1.4 \mathrm{~V}-$ $0.4 \mathrm{~V}$. The maximum frequency variation was only $104 \mathrm{ppm}$ over this span. The measured oscillation frequency was $24 \mathrm{MHz}$ which is suitable for UWB applications. Figure 10 shows the normalized frequency of the different samples as a function of the supply voltage. On the right the center frequency at $1 \mathrm{~V}$ is given for each sample. The measured difference between the oscillation frequencies at $1 \mathrm{~V}$ is $466 \mathrm{kHz}$ or $2 \%$ over the 12 samples. When a higher absolute accuracy is needed, this can be achieved by trimming the center frequency. The oscillator works without any external biasing except for the supply voltage. No trimming or calibration was used to obtain the reported results.

Since the oscillator (including the regulators) has a constant current consumption of $37 \mu \mathrm{A}$, the power consumption decreases proportionally with the supply voltage. If the supply is delivered by a capacitor, the voltage on the capacitor will decrease linearly. The mean power consumption of the oscillator from $1.4 \mathrm{~V}$ to $0.4 \mathrm{~V}$ is then $33 \mu \mathrm{W}$. A separate supply voltage was used for the on-chip output buffers, as these are not needed in a real RFID-tag. In table I, a comparison is made with some state of the art oscillators. Only [5], a high-Q LC oscillator, has a lower voltage-sensitivity. Due to the ultra-low-voltage capabilities, however, the power consumption is the lowest compared to the other designs. Note that this is the only oscillator working in this wide (relative) voltage range.

\section{CONCLUSION}

In this paper a wide-range $0.4-1.4 \mathrm{~V}$ supply-independent oscillator has been presented. An ultra-low-power voltage and current regulator were combined to obtain an oscillation frequency which is strongly independent $(104 \mathrm{ppm} / \mathrm{V})$ of the supply voltage. Due to the use of two coupled oscillators the current consumption of the circuit is equal to $37 \mu \mathrm{A}$ and constant over time. This makes the oscillator suitable for the use on scavenging-based RFIDs as part of a pulsebased UWB transmitter.

\section{ACKNOWLEDGMENT}

The authors acknowledge the financial support of the IWT-SBO program. We also thank Nele Reynders and Jan Verveckken for the useful discussions.

\section{REFERENCES}

[1] C. De Roover and M. Steyaert, "A fully integrated wireless power supply for pinless active rfid-devices in 130nm cmos," Solid-State Circuits Conference, 2007. ASSCC '07. IEEE Asian, pp. 123-126, Nov. 2007.

[2] Z. Li, W. Dehaene, and G. Gielen, "System design for ultra-low-power uwb-based indoor localization," Ultra-Wideband, 2007. ICUWB 2007. IEEE International Conference on, pp. 580-585, Sept. 2007.

[3] M. Verhelst and W. Dehaene, "A flexible, ultra-low-energy 35 pj/pulse digital back-end for a qac ir-uwb receiver," Solid-State Circuits, IEEE Journal of, vol. 43, no. 7, pp. 1677-1687, July 2008.

[4] B. Razavi, "A study of phase noise in CMOS oscillators," Solid-State Circuits, IEEE Journal of, vol. 31, no. 3, pp. 331-343, Mar 1996.

[5] M. S. McCorquodale, J. D. O'Day, S. M. Pernia, G. A. Carichner, S. Kubba, and R. B. Brown, "A monolithic and self-referenced rf lc clock generator compliant with usb 2.0," Solid-State Circuits, IEEE Journal of, vol. 42, no. 2, pp. 385-399, Feb. 2007.

[6] V. De Smedt, P. De Wit, W. Vereecken, and M. Steyaert, "A fullyintegrated Wienbridge topology for ultra-low-power $86 \mathrm{ppm} /{ }^{\circ} \mathrm{C} 65 \mathrm{~nm}$ CMOS 6MHz clock reference with amplitude regulation," 34rd European Solid State Circuits Conference, 2008. ESSCIRC, Sept. 2008.

[7] W. Sansen, Analog Design Essentials. Springer, 2006.

[8] K. P. D'Angelo and R. S. Wrathall, "Compact low dropout voltage regulator using enhancement and depletion mode mos transistors," U.S. Patent 6005378 , Dec. 21, 1999.

[9] Y. Tokunaga, S. Sakiyama, A. Matsumoto, and S. Dosho, "An on-chip cmos relaxation oscillator with power averaging feedback using a reference proportional to supply voltage," Solid-State Circuits Conference, 2009. ISSCC 2009. IEEE International, pp. 404-406, Feb. 2009.

[10] A. Olmos, "A temperature compensated fully trimmable on-chip ic oscillator," Integrated Circuits and Systems Design, 2003. SBCCI 2003. Proceedings. 16th Symposium on, pp. 181-186, Sept. 2003.

[11] K. Sundaresan et al, "Process and temperature compensation in a 7-MHz CMOS clock oscillator," JSSC, vol. 41, no. 2, pp. 433-442, 2006. 